\title{
A metaheuristic to support the distribution of COVID-19 vaccines
}

\author{
Augusto José da Silva Rodrigues ${ }^{\mathrm{a} *}$ (D), Gabriel Lopes Lima ${ }^{\mathrm{a} * *}$ (1) \\ aUniversidade Federal de Pernambuco, Recife, PE, Brasil \\ *augustojsrodrigues@gmail.com**lopeslimagabriel@gmail.com
}

\begin{abstract}
Paper aims: The aim is to develop a vaccine distribution routing model (VDRM) in order to support governments to mitigate the pandemic caused by COVID-19.

Originality: As far as we know, no metaheuristics has been developed for vaccine distribution, and specifically, to support the Brazilian government.
\end{abstract}

Research method: A metaheuristic is developed based on the combination and adaptation of GRASP (Greedy Randomized Adaptive Search Procedure) with VND (Variable Neighborhood Descent), considering different refinement operators. Finally, as a way of validating the model, a numerical application in the state of Pernambuco (Brazil) was performed.

Main findings: Metaheuristic proved to be effective for developing adequate planning for the allocation of ampoules with vaccines to combat COVID-19. Effective analysis was obtained in the evaluation of the proposed algorithm, both in terms of computational effort and the quality of the final solution. An efficiency of approximately $75 \%$ was obtained in relation to the current distribution procedure adopted by the state of Pernambuco.

Implications for theory and practice: To mitigate disease, adequate logistics for transporting and distributing vaccines is essential, especially in emergency situations to face pandemic crises. Thus, the developed metaheuristic can support governments and companies in any situation demanded, making the decision of how the distribution of the ampoules will be more agile.

\section{Keywords}

COVID-19. Vehicle routing. GRASP. VND.

How to cite this article: Rodrigues, A. J. S., \& Lima, G. L. (2021). A metaheuristic to support the distribution of COVID-19 vaccines. Production, 31, e20210031. https://doi.org/10.1590/0103-6513.20210031

Received: Apr. 30, 2021; Accepted: Sep. 17, 2021.

\section{Introduction}

One of the main challenges of the 21 st century is facing the transmission of the new coronavirus (Sars-CoV-2), which triggered the disease named COVID-19, which causes more than 4 million deaths, reported until mid-August 2021 (World Health Organization, 2021). As mentioned by Xiong et al. (2020), the pandemic has drastically altered people's lives, directly impacting the global economy, public and private, in addition to affecting sectors such as tourism, aviation, agriculture and finance.

Due to the above scenario, several organizations have developed or are developing vaccines, as it is probably the most effective approach to sustainably control the COVID-19 pandemic (Koirala et al., 2020). However, it is essential that there is logistical planning for the distribution of these vaccines, as ensuring that they are available reliably to end-users is a major challenge (Yang et al., 2021), especially for poor communities (Petroianu et al., 2020).

Another existing problem is that, in a scenario of vaccine distribution through vehicles, the exact routing models for real problems of great magnitudes require a high computational effort to find solutions (in some cases, taking days), despite providing great results (El-Sherbeny, 2010). Therefore, it is necessary to consider 
the development of models based on heuristics or metaheuristics, which are methods that seek good (almost optimal) solutions at a reasonable computational cost (Rayward-Smith et al., 1996).

Therefore, considering the complexity of vaccine distribution using vehicles, we are faced with the following research question: how to do efficient vaccine distribution? To support governments in decision making, the current paper develops a vaccine distribution routing model (VDRM), based on the construction of a metaheuristic, to assist efficient distribution to society, considering, as a form of validation, a numerical application in the state of Pernambuco, Brazil. At least as far as we know, there is no article that addresses this issue.

\section{Literature review}

\subsection{Vehicle Routing Problem (VRP)}

VRP is a problem where a number of $m$ vehicles must visit a set of $n$ customers to meet their respective demands, and for that, using combined routes to minimize a specific objective, which may be the traveled distance or cost for displacement (Bell \& McMullen, 2004). Thus, VRP is a generalization of the classic Traveling Salesman Problem (TSP) and, consequently, is also characterized as one of the NP-hard problems (Lima et al., 2004).

Mathematically, as established by Laporte (1992), VRP can still be defined as a graph $G=(V, A)$, where $V=(0,1,2 \ldots, n)$ is a set of vertices representing cities, with the deposit (starting point of each vehicle) located at the vertex $V_{0}$ and $A$ is the set of arcs. At each $\operatorname{arc}(i, j)$, given that $i \neq j$, there is an associated non-negative distance matrix $C=\left(C_{i j}\right)$. As mentioned above, it can be interpreted as, for example: travel cost, travel time, trip distance, among others. It depends on the context and the purpose of the decision makers.

There are some conditions for VRP to be satisfied (Mohammed et al., 2017):

- Each vehicle visits a set of customers and returns to the place of departure, that is, the vehicles have their initial and final destinations at the same location (deposit);

- Each location (customer) will be visited once on each route;

- The capacity of each vehicle is sufficient for all applications (meeting all customer demands) included in each route.

The assumptions for VRP can increase and vary, depending on the extent of the problem, which can add time windows (Gendreau \& Tarantilis, 2010), multiple deposits (Montoya-Torres et al., 2015), green vehicles (Lin et al., 2014; Moghdani et al. 2020), periodic vehicles (Campbell \& Wilson, 2014), among others. Other distinct variants are mentioned by Braekers et al. (2016). One of the classic formulations (integer programming) most used for VRP as a basis for several solution methods can be found in Fisher \& Jaikumar (1981).

However, because it is a hard combinatorial optimization problem, VRP in its exact form solves only relatively small instances in an optimized way, which makes it difficult to solve problems as the instances increase, given that exact algorithms based on enumeration partial have a slow convergence rate, which makes, in practice, heuristics more adequate than exact approaches (Cordeau et al., 2002). This is one of the reasons why this paper focuses efforts to develop a metaheuristic, since considering the immensity of the social instance addressed, which are the $n$ cities to receive vaccines against COVID-19, the problem would be impossible to be solved in acceptable computational time, if an exact approach was adopted.

\subsection{GRASP (Greedy Randomized Adaptive Search Procedure)}

The traditional GRASP metaheuristic, originally proposed by Feo \& Resende (1989), is a multi-start algorithm (for successively starting with a distinct "initial solution"), based on two phases: a random construction phase using a function greedy; and an improvement phase to reach a great location (López-Sánchez et al., 2019). A generic GRASP pseudocode is shown in Table 1.

According to GRASP procedure, after reading the problem data, it is necessary to include a rule that establishes the procedure stop criterion, that is, as long as this condition is not met (as a specific number of interactions -GRASPmax) the algorithm remains executed (Sohrabi et al., 2020).

Table 2 shows the constructivist phase of the method, in more detail.

For the constructive stage, it is initially necessary to select a viable solution, consolidated through the interaction between one element at a time, based on a Restricted List of Candidates (RLC) (Ribeiro et al., 2006). This solution will become the current solution. The RLC set is composed of the most interesting elements of 
Table 1. Generic GRASP pseudocode.

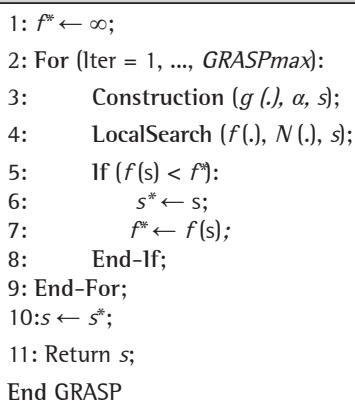

Source: Adapted from Souza (2011).

Table 2. Pseudocode (Construction).

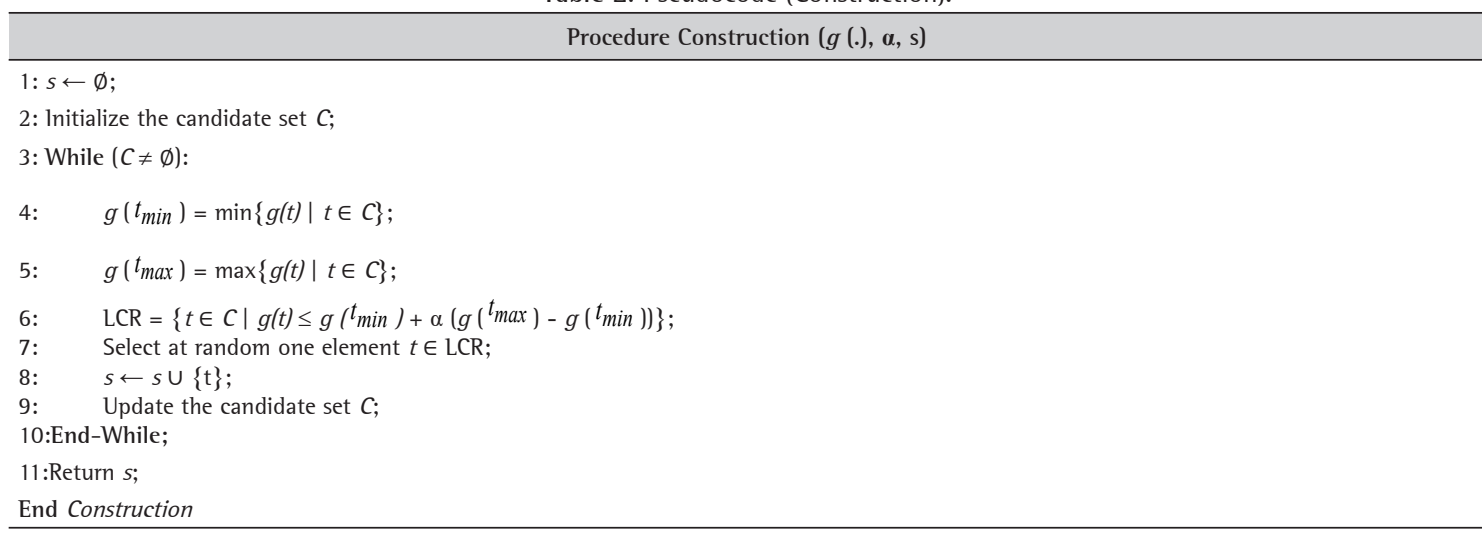

Table 3. Pseudocode (Local Search).

Procedure LocalSearch $(f(),. N(), s$.

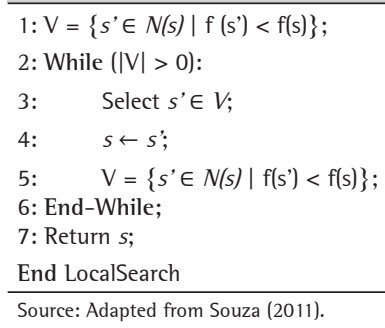

a list of candidates to be included in the solution, following a greedy criterion, and it is exactly this technique that ensures different solutions for each GRASP interaction.

To determine the number of elements in the RLC set, it is necessary to define the value of parameter $\alpha$, which can vary from 0 to 1 . According to López-Sánchez et al. (2019), if $\alpha=0$, the RLC set has only one element and behaves as a constructive heuristic algorithm of greedy type, since if $\alpha=1$, all candidate elements can be part of the RLC set and the choice process becomes totally random. Given the above, there is the second phase of GRASP, which is the phase of local improvement (Table 3).

The local search works as a refinement phase. This phase seeks in the neighborhood of $s$ an improvement in the objective function, and, if found, it is stored. The improvement procedure is performed until a betterperforming neighbor is not found (Cravo \& Amaral, 2019). It is important to note that the local search phase depends on one or more neighborhood operators. Given the end of the algorithm, the solution is defined.

According to Resende \& Ribeiro (2010), the efficiency of the second phase depends on some aspects, such as: a good quality initial construction, a robust neighborhood structure and, mainly, of a strategy used in the 
local improvement phase (e.g., first improvement or best improvement). More details of GRASP can be found in Feo \& Resende (1995) and Resende \& Ribeiro (2019).

\subsection{VND (Variable Neighborhood Descent)}

VND is a local search method proposed by Mladenović \& Hansen (1997), which uses different neighborhood structures/operators to explore the solution space, replacing the current solution with a new one only if there is an improvement (Den Besten \& Stützle, 2001; Jabal-Ameli et al., 2011). As a search strategy, one of the most traditional ones is generally used, namely: "first improvement" or "best improvement" (Hansen et al., 2017). Table 4 presents the pseudocode for the VND.

Table 4. VND pseudocode.

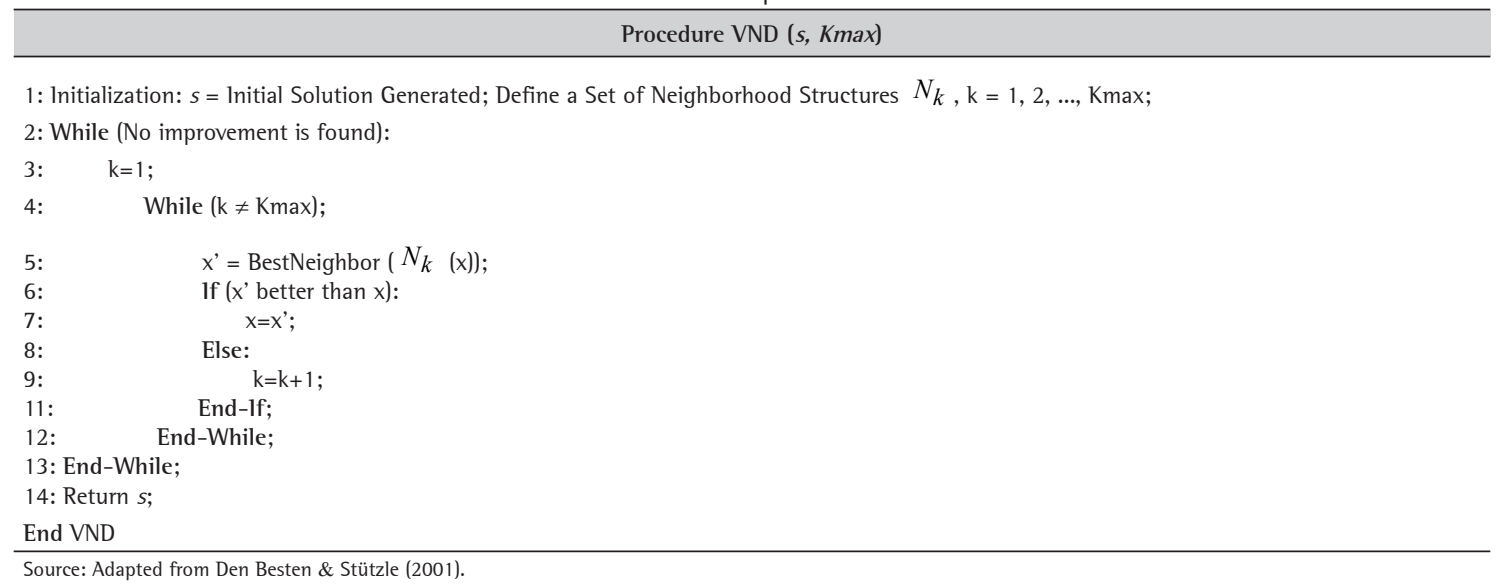

To be considered VND, the algorithm must have at least two neighborhood operators, making it more likely to achieve a global optimum than with a single operator (Hansen et al., 2019). However, depending on the problem addressed, the search for the best neighbor may require a high computational effort, especially if there is a large set of neighborhood structures. Therefore, it is essential for the user to make an analysis of the number of neighborhood structures required.

\section{Methodology}

As the complexity of VRP is NP-Hard, there is no precise algorithm that can quickly solve practical problems with high dimensions. Thus, the use of a metaheuristic presents itself as the most appropriate approach to deal with this type of problem. The methodology for solving VDRM consists of defining the implementation scenario with the instances and the metaheuristic for routing distributions.

\subsection{Scenario}

We consider the state of Pernambuco as a numerical application. It is important to highlight that the model can be applied to other states or even other countries, making the necessary adjustments. Furthermore, we were not successful in contacting the Brazilian government to apply our model. In this sense, our application is only a numerical example. Nevertheless, the data we present here simulate a realistic application, which emphasizes the importance of the study. We intend to persist in contacting the responsible managers in order to apply it in a real case.

According to the Brazilian Ministry of Health (Lara, 2021), vaccines are allocated to Brazilian states through defined flights. Then, each state (also called federative unit) is responsible for the distribution strategies to the municipalities, which can be done in up to seven days.

In Pernambuco, the vaccines arrive in Recife at the Recife/Guararapes - Gilberto Freyre International Airport. Subsequently, the vaccines go to a logistics center that is responsible for forwarding the vaccines to 12 regional 
health managers in the state (GERES). Finally, the secretaries of all municipalities are responsible for picking up the vaccines in each GERES (Pernambuco, 2021).

It is possible to observe that although the state of Pernambuco has 12 GERES, which are cities strategically located throughout the state, there is an ineffective logistical process. Imagine that a municipality is located at point $A$ and its administrative unit at point $B$, for the vaccines to be collected it is necessary to travel twice in the same route $(A \rightarrow B \rightarrow A)$. As it is today in the real case, 172 cities take a duplicate route to go to the 12 GERES.

To improve the abovementioned real case, this research proposes three optimization cases. The first case has Recife as a depot, which receives batches of vaccines via air, with a routing plan containing all the cities in the state departing only from Recife. Cases 2 and 3, on the other hand, consider multiple distribution depots, with Recife and Petrolina in the second case, with the receipt of batches of vaccines also by air, with Recife responsible for the municipalities of GERES located in the east part and Petrolina with the municipalities of the part west. The third case receives batches of vaccines in the 12 central municipalities of GERES, with a routing plan where each central municipality only distributes to the cities belonging to the same GERES.

\subsection{Implementation steps}

The implementation of the algorithm was divided into three steps. In the first step, data were collected from the instances to be applied in VDRM, such as the number of cities, number of vehicles, distances between cities, vehicle capacity, batches of vaccines for distribution and demands of cities. In the second step, GRASP-VND hybrid metaheuristic adjusted for VDRM solution was implemented. In the third stage, computational experiments were carried out.

\subsubsection{Instances}

VDRM has a set of 184 cities that form the supply routes. For the sets of vehicles, cases 1 and 2 have $m=4$, which is the number of vehicles that distribute the vaccines, while case 3 has $m=12$. The batch of vaccines used in the simulation was the same received by the secretariat of the state of Pernambuco on July 2, 2021, with 67,170 doses from the Sinovac/Butantan laboratory, 94,770 doses from Pfizer, 62,250 doses from Janssen and 305,790 doses from AstraZeneca, totalling 529,980 doses of vaccine from COVID-19.

The demands of the cities were defined according to the calculation of the percentage of three indexes in relation to the total of the state for each one of the cities, these indexes are: population number, number of active cases of COVID-19 and number of deaths due to COVID-19 on the same date as the receipt of the vaccine batches. Thus, an average is made between the percentages of these three indexes and from this average percentage the batch of vaccine doses is divided into a balanced way. Additionally, we defined a margin of 5\% for possible cargo damage. When there is a need for reapplication of the vaccine (for example, for a second dose), the metaheuristic should be run again with the respective demands. Figure 1 shows the demand for each municipality in number of doses.

From the balanced proportions of the demands of the cities, the problem is their safe transport, for this the Ministry of Health's cold chain manual (Brasil, 2017) defines the guidelines for equipment, storage and

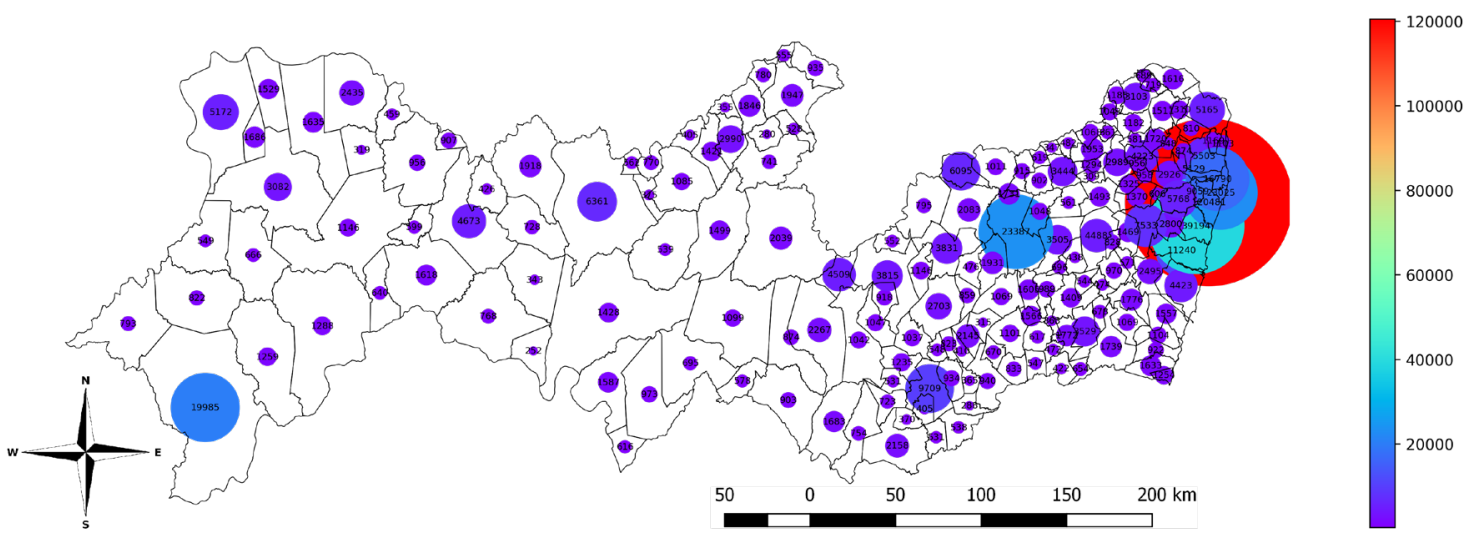

Figure 1. Density of vaccine demand by city. 
transport of immunobiologicals, in which it is established that for the transport of vaccines the road modal with refrigerated vehicles is used.

Immunobiologicals are packaged in expanded polystyrene coolers with recyclable ice coils on the bottom and side walls of the box. Thus, vehicles for transporting refrigerated immunobiologicals have the capacity to transport 24 coolers, each cooler has the capacity of 10 vials of 60 units, and each vial has 10 doses of the vaccine, so the vehicles have the capacity to safely transport 144,000 doses.

To obtain the distances, the map of Pernambuco (Instituto Brasileiro de Geografia e Estatistica, 2019) was processed in the QGIS software, which is an open-source geographic information system (GIS) (QGIS, 2021). Through the QGIS vector commands, 184 points were obtained, with coordinates representing each municipality in the state, not considering the island of Fernando de Noronha. To form the distance matrices between regions, geometric distances in multidimensional space, known as Euclidean distances, were calculated.

\subsection{Exact formulation of the VDRM}

To formulate the exact solution of the vaccine distribution routing model it is necessary to review and adapt classical formulations Multi-Depot Vehicle Routing Problem (MDVRP) (Kulkarni \& Bhave, 1985; MontoyaTorres et al., 2015).

The mathematical model of the MDVRP is defined by Renaud et al. (1996) as a graph $G=(V, E)$ where $V$ is the set of nodes and $E$ is the set of arcs or edges connecting each pair of nodes. Furthermore, $V$ consists of two subsets: the set of cities or customers $\left(V_{c}=\left\{v_{1}, \ldots, v_{N}\right\}\right)$ and the set of deposits $\left(V_{d}=\left\{v_{N+1}, \ldots, v_{M}\right\}\right)$. Moreover, each customer ${ }^{v_{i}}$ has a nonnegative demand $d_{i}$.

Thus, the problem consists of minimizing the traveled distance $c_{i j}$, according to Equation 1 , where $K$ represents the total number of vehicles and ${ }^{x_{i j k}}$ the binary decision variables, which can equal 1 if the pair of nodes $i$ and $j$ are in the route of vehicle $k$, or 0 otherwise.

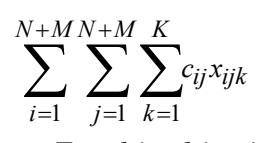

For this objective, there are nine indispensable constraints. The first two constraints (2 and 3) must ensure that each customer is served by one and only one vehicle.

$$
\sum_{i=1}^{N+M} \sum_{k=1}^{K} x_{i j k}=1 \quad \forall j=1, \ldots, N
$$

$$
\sum_{j=1}^{N+M} \sum_{k=1}^{K} x_{i j k}=1 \quad \forall \quad i=1, \ldots, N
$$

Route continuity is preserved by constraint 4 .

$$
\sum_{i=1}^{N+M} x_{i h k}-\sum_{j=1}^{N+M} x_{h j k}=0 \quad \forall k=1, \ldots, K, h=1, \ldots, N+M
$$

Our contribution is in constraint 5 , which defines the percentage of vaccines demanded by each city in a specified territory (e.g., state or country). In this constraint, $p_{i}, \gamma_{i}$ and $\sigma_{i}$ represent the population number, number of active cases of COVID-19 and number of deaths due to COVID-19 for each city, respectively. This means that $p_{\text {total }}, \gamma_{\text {total }}$ and $\sigma_{\text {total }}$ represent these same indices for the total territory (e.g., Pernambuco State, Brazil). Finally, $\varphi_{i}$ represents the number of available vaccines of each type, where $\Phi$ represents the set of vaccines.

$$
Q_{i}=\frac{\frac{p_{i}}{p_{\text {total }}}+\frac{\gamma_{i}}{\gamma_{\text {total }}}+\frac{\sigma_{i}}{\sigma_{\text {total }}}}{3} * \sum_{i=1}^{\Phi} \varphi_{i} \quad \forall i=1, \ldots, N
$$


Each vehicle’s capacity, $P_{k}$, must be guaranteed, as specified in constraint 6 .

$$
\sum_{i=1}^{N+M} Q_{i} \sum_{j=1}^{N+M} x_{i j k} \leq P_{k} \quad \forall k=1, \ldots, K
$$

Vehicle availability is verified by constraints 7 and 8 .

$$
\begin{aligned}
& \sum_{i=N+1}^{N+M} \sum_{j=1}^{N} x_{i j k} \leq 1 \forall k=1, \ldots, K \\
& \sum_{j=N+1}^{N+M} \sum_{i=1}^{N} x_{i j k} \leq 1 \forall k=1, \ldots, K
\end{aligned}
$$

Subtour elimination is provided by constraint 9 .

$$
y_{i}-y_{j}+(M+N) \sum_{k=1}^{V} x_{i j k} \leq N+M-1, \text { for } i \leq i \neq j \leq M+N-1
$$

The last constraint (10) ensures that the decision variables are binary.

$x_{i j k} \in\{0,1\} \forall i, j, k$

Since this formulation is an extension of the classical VRP presented in subsection 2.1, it is also an NP-hard problem. Therefore, it is impossible to solve this exact formulation in an acceptable computational time, which justifies the metaheuristics developed in the next section.

\subsection{Metaheuristic GRASP-VND}

The proposed metaheuristic is a Greedy Randomized Adaptive Search Procedure (GRASP). This metaheuristic uses in its construction stage the classic Restricted Candidate list (RCL) and an $\alpha$ parameter that controls the greedy and random approach of the process. Thus, in a way that the capacity of the vehicles is respected, the procedure ends when all municipalities are on a route. In the local search stage, the Variable Neighborhood Descent (VND) refinement method was used. The pseudocode of the implemented metaheuristics for the definition of routes is shown in Table 5.

Thus, from the instances received by the algorithm, line 3 of the pseudocode shows that a series of iterations was executed, wherein each iteration an initial route was generated for each vehicle (line 4), thus determining which cities were selected for the route through a list of candidates $C$ (line 5). This list follows a logic that respects restrictions 2,3 , and 4 , which ensure that each city is visited only once by only one vehicle and that every vehicle that visits a city delivering vaccine batches withdraws from it to the next city or the origin, ensuring continuity.

For the number of cities that make up the route of each vehicle, restrictions 5, 6, 7, and 8 are respected, where each vehicle supports a number of cities that do not exceed its capacity and availability. In the local search phase, different routes were defined according to the best neighbor in the sets of routes in the neighborhoods of the different operators, thus seeking to minimize the total distance of the routes, fulfilling the objective function.

The use of VND makes the algorithm capable of exploring different solution spaces with the sequential use of neighborhood operators, who seek to effect exchange changes between the sets of destinations present in the current solution. Eleven operators were used, five of which were to improve the route of each vehicle, namely or-opt1, or-opt2 and or-opt3, which consists of removing one, two or three adjacent cities for insertion in another position. Swap, where the exchange is made between two non-adjacent cities and 2-opt that removes two arcs from a route and inserts two new arcs at the same route, and six operators for improvement between the vehicle routes, which are shift( $(1,0)$, shift $(2,0)$, shift $(3,0)$, that consists of transferring one, two or three cities from one route to another route, finally the $\operatorname{swap}(1,1), \operatorname{swap}(2,2)$ and $\operatorname{swap}(3,3)$, which consists of exchanging one, two or three cities on one route with cities on another route. 
Table 5. GRASP + VND pseudocode.

Algorithm GRASP + VND (Matrix of distances, demands, capacity, $\boldsymbol{\alpha}$, itermax)

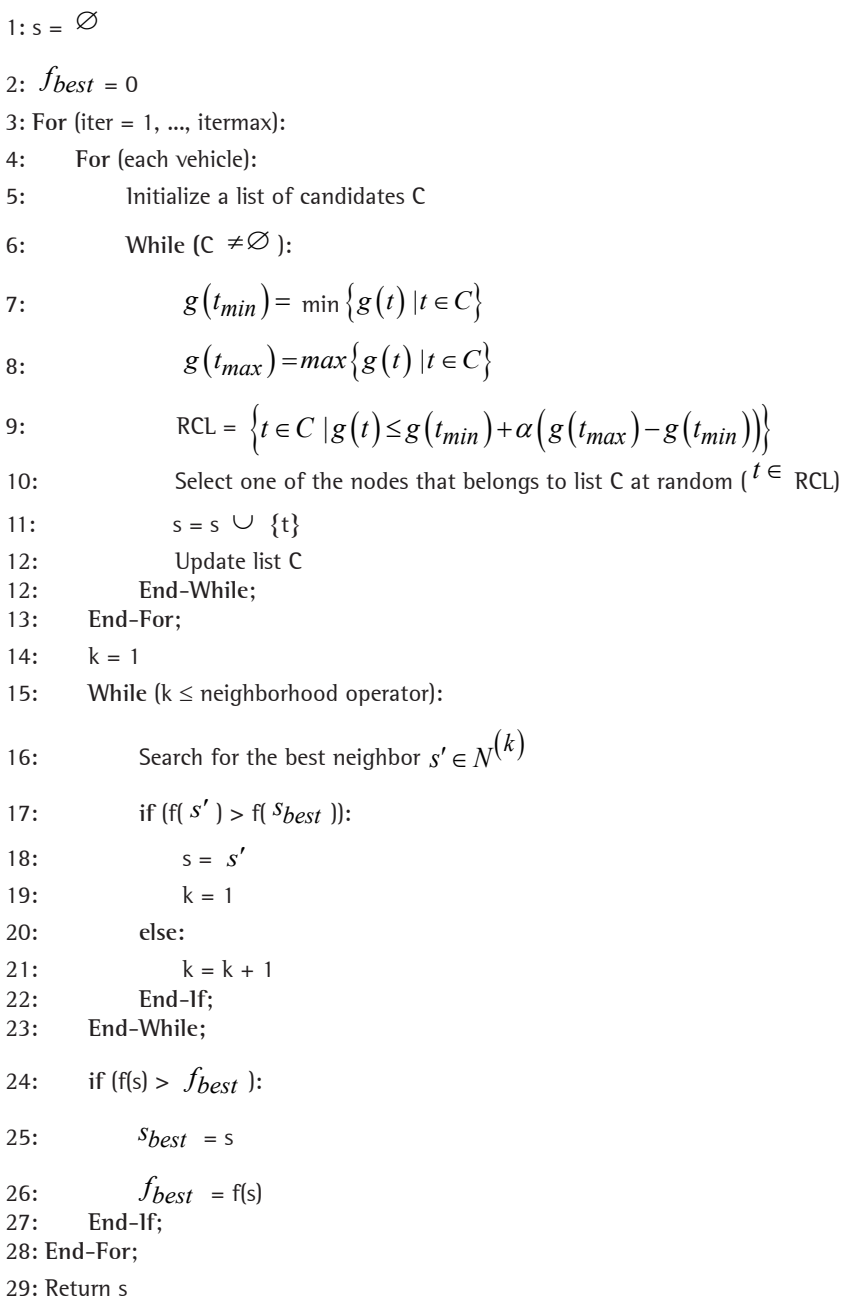

\section{Results and discussions}

The proposed metaheuristic was implemented in Python 3 and executed on a computer with an Intel Core i7 processor, $8 \mathrm{~GB}$ of installed memory (RAM), 64bit windows 10 operating system. With the instances collected and generated based on the 184 municipalities of the state of Pernambuco, with three different optimization cases for comparison with the real case, GRASP-VND metaheuristic for each case was executed 30 times with the best adaptation alpha for each case, the best solution being considered for analysis. Shown in Table 6 the values of best, mean and standard deviation found for the distances in $\mathrm{km}$ and the computational execution time of the algorithm in seconds.

Table 6. GRASP-VND metaheuristic results.

\begin{tabular}{|c|c|c|c|c|c|}
\hline & Case & Real & 1 & 2 & 3 \\
\hline \multirow{3}{*}{ Distance $(\mathrm{km})$} & Best & 14,434 & 3,974 & 3,513 & 3,655 \\
\hline & Average & - & $4,320.55$ & $3,704.43$ & $3,662.27$ \\
\hline & Standard deviation & - & 211.06 & 94.01 & 8.48 \\
\hline \multirow{3}{*}{ Time (s) } & Best & 0.001 & $1,050.36$ & 206.78 & 22.26 \\
\hline & Average & - & $2,870.11$ & 384.49 & 37.35 \\
\hline & Standard deviation & - & 971.65 & 108.36 & 9.94 \\
\hline
\end{tabular}


The best optimization to minimize the distances traveled in the distribution of vaccines is the second case, which has Recife and Petrolina as distribution depots. This case had a total distance of 3,513 km traveled by 4 vehicles, a saving of almost $11,000 \mathrm{~km}$ compared to the real case, thus representing a routing $75.66 \%$ more efficient than that currently performed by the state health department. The third and first cases follow as good alternatives with 3,655 and 3,974 $\mathrm{km}$ respectively. However, cases 1 and 2 have high standard deviation values with 211 and 94, respectively, this dispersion is better visualized in the boxplot graphs in Figure 2.

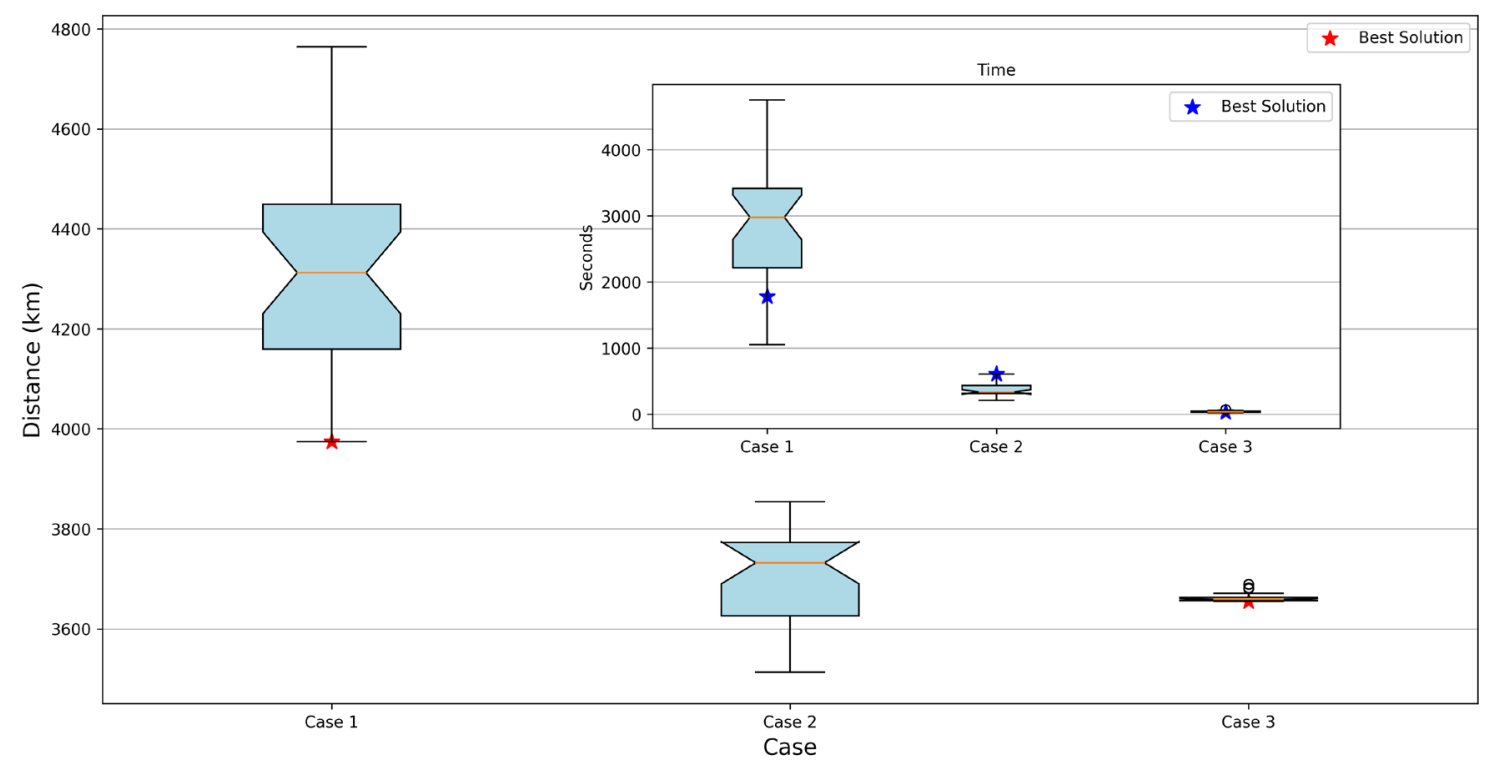

Figure 2. Boxplot graph of the distance and time results of the algorithm.

Analyzing the dispersions of these cases, case 3 presents values well concentrated in the average of $3,662 \mathrm{~km}$, with a standard deviation of only $8.48 \mathrm{~km}$. This low interquartile range, is due to less complexity for the optimization of this case, considering that it is only one vehicle is used to route the cities of GERES itself, thus reducing $n$ from 184 to the number of cities in each GERES. Cases 1 and 2, on the other hand, have greater interquartile ranges, a natural factor because as the number of cities $n$ increases, all 184 in case 1 and the division of east and west cities in case 2, there is an increase in complexity of the model, as they are considered new distance analyses. Time also goes through this increase, because the more cities to be considered, the greater the possibilities for searching in the neighborhood, with the selection of the best improvement, which makes the local search even longer, but with the best possible result.

These higher interquartile variability and standard deviations are due to GRASP-VND method being a metaheuristic that works with randomness, with the possibility of different results running the same algorithm, mainly due to the diversification in the generation of initial solutions. The real case route and the optimized case routes can be analyzed from Figure 3.

From the results obtained, it is evident that some vehicles will travel more cities (and consequently, a greater distance) than other vehicles. This is due to the variation in demands around the state, where generally there are sets of cities that return a sum of demands many times lower than the demand of a single city, for example. In this case, the behavior referred to is expected, given that the objective of the model is to minimize the distance covered, considering the aggregation of the distances of the four vehicles. The scenario would change, possibly, if it were considered to minimize the time traveled by vehicles.

\section{Final considerations}

The objective of the paper was concluded, since it was possible to provide vehicle routing for distributing vaccines against COVID-19, considering the construction of a metaheuristic, given the difficulty of the problem addressed. The metaheuristic showed an efficiency of $75.66 \%$ in relation to the current way of distribution 

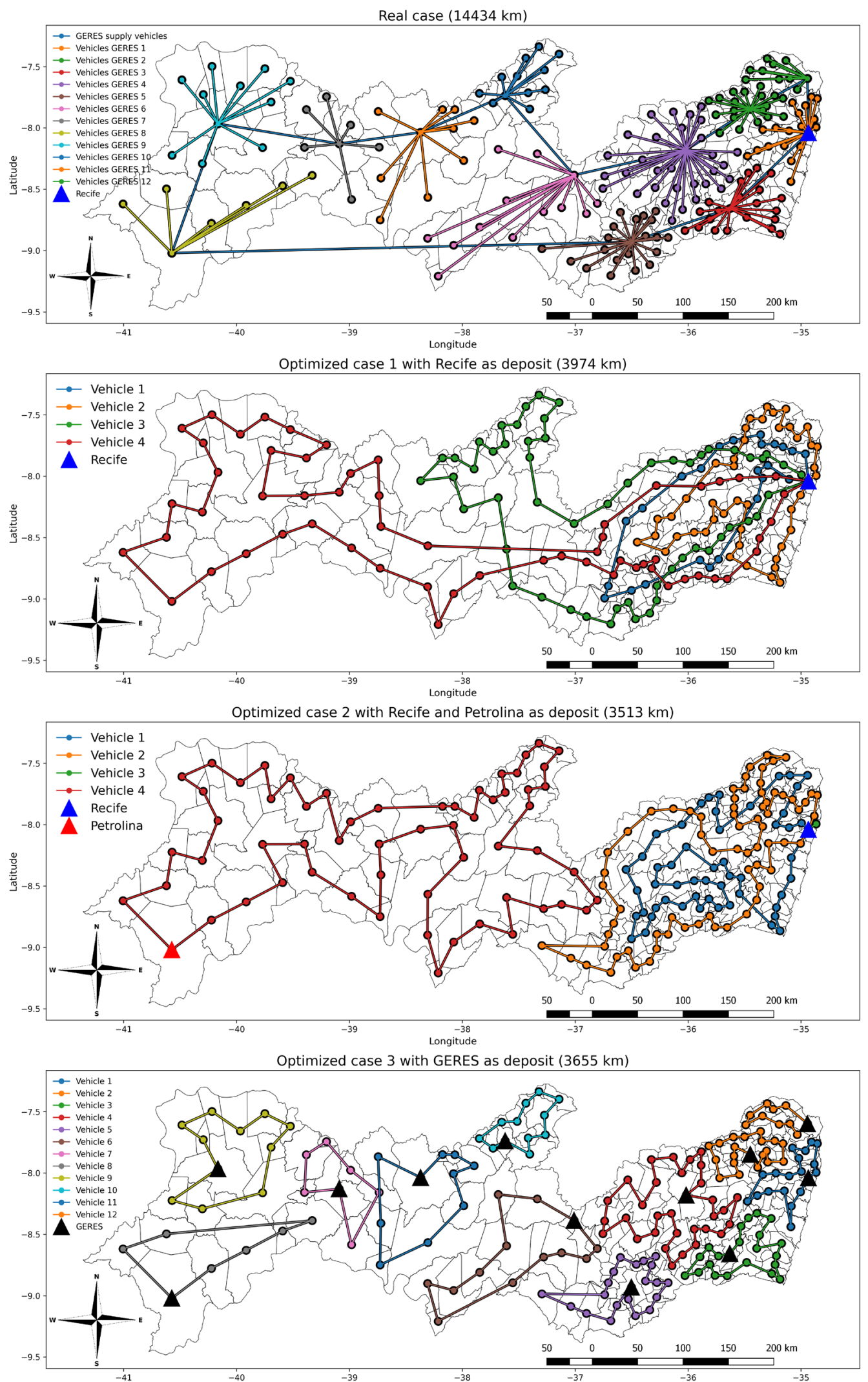

Figure 3. Plots of real case and optimized case routes. 
considered by the state of Pernambuco. Additionally, although the application and validation of the model were based on the state of Pernambuco (Brazil), it is possible to extend the model to other states, regions or even a country.

More specifically, our metaheuristics can be used in multiple ways for routing vehicles that distribute other ampoules to combat other types of diseases. The reference to the use of multiple ways, that is to say: depending on the need to deliver the vaccines, it is possible to increase the number of vehicles available, to reduce the delivery time (when there is urgency), or to reduce the number of vehicles (when there is no urgency), in order to generate cost savings. In the latter case, it is necessary to carry out a financial analysis to understand the feasibility of the scenario.

One difficulty of this study was to compare the performance of the proposed metaheuristics with other models, as it was not possible to find an exact algorithm that solved instances of the same size (returning the optimum value), for the same problem. Nevertheless, the execution of GRASP-VND of this study managed to solve most instances in terms of seconds, which already satisfies the purpose of this paper.

Given the above, the importance of developing works for vehicle routing aiming at the distribution of vaccines, even before its approval, is highly relevant, since logistical operations are full of challenges and need, as a way to mitigate the impacts of the pandemic, agility in planning the entire supply chain.

\subsection{Suggestions for future research}

The paper considered identical items (identical volumes), as it illustrates the distribution of ampoules only to combat COVID-19. In addition, the number of vehicles required for routing was established based on the demands of the cities and the volume of all trucks. However, when there is a need to distribute ampoules for different diseases (with different volumes), it is crucial to establish the best way to conduct the packaging inside the trucks. For this reason, it is suggested to aggregate the packaging problem in the proposed metaheuristic, as this would make it possible to perform vehicle routing with ampoules for different diseases, minimizing the number of vehicles and containers used.

In another view, it is advisable to build other metaheuristics (like Tabu Search, Variable Neighborhood Search, Simulating Annealing and Genetic Algorithm) to compare performances, both of the computational effort and of the quality of the final solution. Moreover, a cost evaluation should be added, considering costs such as: fuel, cooling, penalties, and so on. Additionally, addressing the time window constraint is fundamental, in order to respect a daily operation limit. Finally, it is necessary to restrict delivery to commercial hours and to consider that a heterogeneous fleet of vehicles, as there is a difference in car models in each city.

\section{References}

Bell, J. E., \& McMullen, P. R. (2004). Ant colony optimization techniques for the vehicle routing problem. Advanced Engineering Informatics, 18(1), 41-48. http://dx.doi.org/10.1016/j.aei.2004.07.001.

Braekers, K., Ramaekers, K., \& Van Nieuwenhuyse, 1. (2016). The vehicle routing problem: State of the art classification and review. Computers \& Industrial Engineering, 99, 300-313. http://dx.doi.org/10.1016/j.cie.2015.12.007.

Brasil. Ministério da Saúde. (2017). Manual de Rede de Frio do Programa Nacional de Imunizações. Retrieved in 2021, April 30, from https://portalarquivos2.saude.gov.br/images/pdf/2017/dezembro/15/rede_frio_2017_web_VF.pdf

Campbell, A. M., \& Wilson, J. H. (2014). Forty years of periodic vehicle routing. Networks, 63(1), 2-15. http://dx.doi.org/10.1002/net.21527.

Cordeau, J. F., Gendreau, M., Laporte, G., Potvin, J. Y., \& Semet, F. (2002). A guide to vehicle routing heuristics. The Journal of the Operational Research Society, 53(5), 512-522. http://dx.doi.org/10.1057/palgrave.jors.2601319.

Cravo, G. L., \& Amaral, A. R. (2019). A GRASP algorithm for solving large-scale single row facility layout problems. Computers \& Operations Research, 106, 49-61. http://dx.doi.org/10.1016/j.cor.2019.02.009.

Den Besten, M., \& Stützle, T. (2001). Neighborhoods revisited: an experimental investigation into the effectiveness of variable neighborhood descent for scheduling. In Proceedings of the MIC'2001-4th Metaheuristics International Conference (pp. 545-549). Porto, Portugal.

El-Sherbeny, N. A. (2010). Vehicle routing with time windows: an overview of exact, heuristic and metaheuristic methods. Journal of King Saud University-Science, 22(3), 123-131. http://dx.doi.org/10.1016/j.jksus.2010.03.002.

Feo, T. A., \& Resende, M. G. (1989). A probabilistic heuristic for a computationally difficult set covering problem. Operations Research Letters, 8(2), 67-71. http://dx.doi.org/10.1016/0167-6377(89)90002-3.

Feo, T. A., \& Resende, M. G. (1995). Greedy randomized adaptive search procedures. Journal of Global Optimization, 6(2), $109-133$. http://dx.doi.org/10.1007/BF01096763.

Fisher, M. L., \& Jaikumar, R. (1981). A generalized assignment heuristic for vehicle routing. Networks, 11(2), 109-124. http://dx.doi. org/10.1002/net.3230110205.

Gendreau, M., \& Tarantilis, C. D. (2010). Solving large-scale vehicle routing problems with time windows: the state-of-the-art. Montreal: Cirrelt. 
Hansen, P., Mladenović, N., Brimberg, J., \& Pérez, J. A. M. (2019). Variable neighborhood search. In M. Gendreau \& J. Y. Potvin (Eds.), Handbook of metaheuristics (pp. 57-97). Cham: Springer. http://dx.doi.org/10.1007/978-3-319-91086-4_3.

Hansen, P., Mladenović, N., Todosijević, R., \& Hanafi, S. (2017). Variable neighborhood search: basics and variants. EURO Journal on Computational Optimization, 5(3), 423-454. http://dx.doi.org/10.1007/s13675-016-0075-x.

Instituto Brasileiro de Geografia e Estatistica - IBGE. (2019). IBGE MAPS portal. Retrieved in 2021, April 12, from https://portaldemapas. ibge.gov.br/portal.php\#homepage

Jabal-Ameli, M. S., Aryanezhad, M. B., \& Ghaffari-Nasab, N. (2011). A variable neighborhood descent based heuristic to solve the capacitated location-routing problem. International Journal of Industrial Engineering Computations, 2(1), 141-154. http://dx.doi. org/10.5267/j.ijiec.2010.06.003.

Koirala, A., Joo, Y. J., Khatami, A., Chiu, C., \& Britton, P. N. (2020). Vaccines for COVID-19: The current state of play. Paediatric Respiratory Reviews, 35, 43-49. http://dx.doi.org/10.1016/j.prrv.2020.06.010. PMid:32653463.

Kulkarni, R. V., \& Bhave, P. R. (1985). Integer programming formulations of vehicle routing problems. European Journal of Operational Research, 20(1), 58-67. http://dx.doi.org/10.1016/0377-2217(85)90284-X.

Laporte, G. (1992). The vehicle routing problem: An overview of exact and approximate algorithms. European Journal of Operational Research, 59(3), 345-358. http://dx.doi.org/10.1016/0377-2217(92)90192-C.

Lara, M. A. (2021, July 5). Saiba como é realizada a distribuição da vacina Covid-19 para os estados. Brasília: Ministério da Saúde. Retrieved in 2021, April 12, from https://www.gov.br/saude/pt-br/assuntos/noticias/saiba-como-e-realizada-a-distribuicao-davacina-covid-19-para-os-estados

Lima, C. D. R., Goldbarg, M. C., \& Goldbarg, E. F. G. (2004). A memetic algorithm for the heterogeneous fleet vehicle routing problem. Electronic Notes in Discrete Mathematics, 18, 171-176. http://dx.doi.org/10.1016/j.endm.2004.06.027.

Lin, C., Choy, K. L., Ho, G. T., Chung, S. H., \& Lam, H. Y. (2014). Survey of green vehicle routing problem: past and future trends. Expert Systems with Applications, 41(4), 1118-1138. http://dx.doi.org/10.1016/j.eswa.2013.07.107.

López-Sánchez, A. D., Sánchez-Oro, J., \& Hernández-Díaz, A. G. (2019). GRASP and VNS for solving the p-next center problem. Computers \& Operations Research, 104, 295-303. http://dx.doi.org/10.1016/j.cor.2018.12.017.

Mladenović, N., \& Hansen, P. (1997). Variable neighborhood search. Computers \& Operations Research, 24(11), 1097-1100. http:// dx.doi.org/10.1016/S0305-0548(97)00031-2.

Moghdani, R., Salimifard, K., Demir, E., \& Benyettou, A. (2020). The green vehicle routing problem: A systematic literature review. Journal of Cleaner Production, 123691. http://dx.doi.org/10.1016/j.jclepro.2020.123691.

Mohammed, M. A., Ghani, M. K. A., Hamed, R. 1., Mostafa, S. A., lbrahim, D. A., Jameel, H. K., \& Alallah, A. H. (2017). Solving vehicle routing problem by using improved K-nearest neighbor algorithm for best solution. Journal of Computational Science, 21, 232-240. http://dx.doi.org/10.1016/j.jocs.2017.04.012.

Montoya-Torres, J. R., Franco, J. L., Isaza, S. N., Jiménez, H. F., \& Herazo-Padilla, N. (2015). A literature review on the vehicle routing problem with multiple depots. Computers \& Industrial Engineering, 79, 115-129. http://dx.doi.org/10.1016/j.cie.2014.10.029.

Pernambuco. Governo do Estado. Secretaria da Saúde. (2021, July 7). Governo de Pernambuco conclui distribuição de mais 110.800 doses de vacina a todos os municipios. Retrieved in 2021, April 12, from https://www.pecontracoronavirus.pe.gov.br/governo-depernambuco-conclui-distribuicao-de-mais-110-800-doses-de-vacina-a-todos-os-municipios/

Petroianu, L. P. G., Zabinskya, Z. B., Zameer, M., Chu, Y., Muteia, M. M., Resende, M. G. C., Coelho, A. L., Wei, J., Purty, T., Draiva, A., \& Lopes, A. (2020). A light-touch routing optimization tool (RoOT) for vaccine and medical supply distribution in Mozambique. International transactions in operational research : a journal of The International Federation of Operational Research Societies, 28(5), 2334-2358. http://dx.doi.org/10.1111/itor.12867. PMid:33883827.

QGIS. (2021, April 12). A Free and Open Source Geographic Information System. Retrieved in 2021, April 12, from https://www.qgis. org/en/site/index.html

Rayward-Smith, V. J., Osman, l. H., Reeves, C. R., \& Smith, G. D. (1996). Modern heuristic search methods. Hoboken: Wiley.

Renaud, J., Laporte, G., \& Boctor, F. F. (1996). A tabu search heuristic for the multi-depot vehicle routing problem. Computers \& Operations Research, 23(3), 229-235. http://dx.doi.org/10.1016/0305-0548(95)00026-P.

Resende, M. G., \& Ribeiro, C. C. (2010). Greedy randomized adaptive search procedures: advances, hybridizations, and applications. In M. Gendreau \& J. Y. Potvin (Eds.), Handbook of metaheuristics (pp. 283-319). Boston, MA: Springer. http://dx.doi.org/10.1007/9781-4419-1665-5_10.

Resende, M. G., \& Ribeiro, C. C. (2019). Greedy randomized adaptive search procedures: advances and extensions. In M. Gendreau \& J. Y. Potvin (Eds.), Handbook of metaheuristics (pp. 169-220). Cham: Springer. http://dx.doi.org/10.1007/978-3-319-91086-4_6.

Ribeiro, M. H., Plastino, A., \& Martins, S. L. (2006). Hybridization of GRASP metaheuristic with data mining techniques. Journal of Mathematical Modelling and Algorithms, 5(1), 23-41. http://dx.doi.org/10.1007/s10852-005-9030-1.

Sohrabi, S., Ziarati, K., \& Keshtkaran, M. (2020). A greedy randomized adaptive search procedure for the orienteering problem with hotel selection. European Journal of Operational Research, 283(2), 426-440. http://dx.doi.org/10.1016/j.ejor.2019.11.010.

Souza, M. J. F. (2011). Inteligência Computacional para Otimização. Universidade Federal de Ouro Preto. Retrieved in 2021, April 12, from http://www.decom.ufop.br/prof/marcone/Disciplinas/InteligenciaComputacional/InteligenciaComputacional.pdf

World Health Organization - WHO. (2021). WHO Coronavirus (COVID-19) Dashboard. Retrieved in 2021, April 12, from https://covid19. who.int/

Xiong, J., Lipsitz, O., Nasri, F., Lui, L. M. W., Gill, H., Phan, L., Chen-Li, D., lacobucci, M., Ho, R., Majeed, A., \& Mclntyre, R. S. (2020). Impact of COVID-19 pandemic on mental health in the general population: a systematic review. Journal of Affective Disorders, 277, 55-64. http://dx.doi.org/10.1016/j.jad.2020.08.001. PMid:32799105.

Yang, Y., Bidkhori, H., \& Rajgopal, J. (2021). Optimizing vaccine distribution networks in low and middle-income countries. Omega, 99, 102197. http://dx.doi.org/10.1016/j.omega.2020.102197. 